\section{Commentary: A new hope: Do ADAURA trial results change the paradigm for treatment of resectable lung adenocarcinoma?}

\author{
Sue Xue Wang, MD, and M. Blair Marshall, MD
}

The advent of targeted therapy for oncologic drivers in the treatment of lung adenocarcinoma (LUAD) has the potential to propel the field into hyperspace. Jones and colleagues ${ }^{1}$ have presented a well-crafted and optimistic review of epidermal growth factor receptor (EGFR) tyrosine kinase inhibitors - their past, present, and future. As the third iteration of EGFR- tyrosine kinase inhibitors, osimertinib has demonstrated superior efficacy and safety compared with gefitinib or erlotinib. ${ }^{2}$ ADAURA randomized patients with completely resected IB to IIIA EGFRvariant positive $(\mathrm{EGFRm}+)$ LUAD to 3 years of adjuvant osimertinib versus placebo. ${ }^{3}$ Osimertinib was found to have dramatic benefits in disease-free survival (DFS); however, these benefits are more ambiguous than they appear at first glance.

The authors contrast the modest benefits of adjuvant chemotherapy with the striking potential benefits of adjuvant osimertinib. The Lung Adjuvant Cisplatin Evaluation trial demonstrated improved overall survival with adjuvant chemotherapy, where the overall hazard ratio $(\mathrm{HR})$ of death was $0.89 \quad(95 \% \quad \mathrm{CI}, \quad 0.82-0.96$; $P=.005)$. This correlates with a 5-year benefit of $5.4 \%{ }^{4}$ In contrast, in ADAURA, overall DSF at 24 months was $90 \%$ with osimertinib versus $44 \%$ with placebo (overall HR, 0.17; 99\% CI, 0.11-0.26) for patients with stage II to IIIA LUAD. ADAURA presents an $83 \%$ reduction in disease recurrence and death, a

\footnotetext{
From the Division of Thoracic Surgery, Department of Surgery, Brigham and Women's Hospital, Boston, Mass.

Disclosures: The authors reported no conflicts of interest.

The Journal policy requires editors and reviewers to disclose conflicts of interest and to decline handling or reviewing manuscripts for which they may have a conflict of interest. The editors and reviewers of this article have no conflicts of interest.

Received for publication Feb 18, 2021; revisions received Feb 18, 2021; accepted for publication Feb 18, 2021; available ahead of print Feb 26, 2021.

Address for reprints: M. Blair Marshall, MD, Division of Thoracic Surgery, Department of Surgery, Brigham and Women's Hospital, 75 Francis St, Boston, MA 02115 (E-mail: mmarshall16@bwh.harvard.edu).

J Thorac Cardiovasc Surg 2021;162:293-4 $0022-5223 / \$ 36.00$

Copyright (c) 2021 Published by Elsevier Inc. on behalf of The American Association for Thoracic Surgery

http://dx.doi.org/10.1016/j.jtcvs.2021.02.073
}

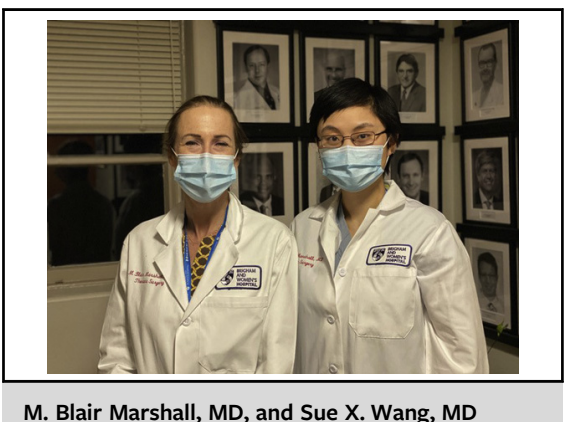

M. Blair Marshall, MD, and Sue X. Wang, MD

$$
\begin{aligned}
& \text { CENTRAL MESSAGE } \\
& \text { Longer follow-up data from the } \\
& \text { ADAURA trial are required to } \\
& \text { determine whether osimertinib is } \\
& \text { associated with improved overall } \\
& \text { survival before adopting targeted } \\
& \text { therapy in adjuvant settings. }
\end{aligned}
$$

benefit that far outstrips the current standard of care and inspires hope for a new era in the treatment of resectable LUAD.

Nonetheless, we temper our optimism at the promise of osimertinib with caution. ADAURA raises many clinical and economical questions. Although half of study participants were randomized to receive daily osimertinib for 3 years, applying this regimen in practice carries a prohibitive cost of more than $\$ 200,000$ per patient per year in the United States. ${ }^{5}$ The authors acknowledge the chief limitation of ADAURA-we do not know if adjuvant osimertinib improves overall survival. Other randomized trials, including Gefitinib Versus Vinorelbine Plus Cisplatin as Adjuvant Treatment For Stage II-IIIA (N1-N2) EGFR-Mutant Non-Small Cell Lung Cancer (ADJUVANT/CTONG1104) $^{6}$ and Erlotinib Versus Chemotherapy as First Line Treatment of EGFT Mutation-Positive Advanced Non-Small Cell Lung Cancer (OPTIMAL) $)^{7}$ have failed to show improvements in DFS correlating with overall survival. Is the great financial burden of osimertinib worth an unclear survival advantage?

What are the implications of ADAURA on patients with lower-risk, stage IB LUAD? Because thoracic surgeons are primarily responsible for the treatment of early-stage LUAD, we will be faced with the decision to refer patients for standard adjuvant chemotherapy or to targeted adjuvant therapy based on tumor genetic profiling. The ADAURA subset of stage IB patients demonstrated a much less remarkable HR of 0.5 compared with 0.17 in stage II or 
IIIA LUAD. We find it disconcerting that only $60 \%$ of patients in ADAURA received adjuvant chemotherapy and note an unusually high rate of recurrence in the placebo $\mathrm{arm}$. There are too many unknowns to disregard the proven survival advantage of adjuvant chemotherapy for the unproven benefit of osimertinib.

Longer follow-up is required to see if ADAURA endures the test of time and adjuvant osimertinib confers a survival advantage. Regardless, we are certain that there is an imminent and inevitable paradigm shift toward targeted therapy in the treatment of resectable LUAD on the horizon.

\section{References}

1. Jones D, Wu YL, Tsuboi M, Herbst R. Targeted therapies for resectable lung adenocarcinoma: ADAURA opens for thoracic oncologic surgeons. J Thorac Cardiovasc Surg. 2021;162:288-92.
2. Ramalingam SS, Vansteenkiste J, Planchard D, Cho BC, Gray JE, Ohe Y, et al. Overall survival with osimertinib in untreated, EGFR-mutated advanced NSCLC. N Engl J Med. 2020;382:41-50.

3. Wu Y-L, Tsuboi M, He J, John T, Grohe C, Majem M, et al. Osimertinib in resected EGFR-mutated non-small-cell lung cancer. $N$ Engl J Med. 2020;383: 1711-23.

4. Pignon JP, Tribodet H, Scagliotti GV, Douillard JY, Shepherd FA, Stephens RJ, et al. Lung adjuvant cisplatin evaluation: a pooled analysis by the LACE Collaborative Group. J Clin Oncol. 2008;26:3552-9.

5. West H, Gyawali B. Why not adore ADAURA? - The trial we need vs the trial we got. JAMA Oncol. February 4, 2021 [Epub ahead of print].

6. Zhong WZ, Wang Q, Mao W-M, Xu ST, Wu L, Shen Y, et al. Gefitinib versus vinorelbine plus cisplatin as adjuvant treatment for stage II-IIIA (N1-N2) EGFRmutant NSCLC (ADJUVANT/CTONG1104): a randomised, open-label, phase 3 study. Lancet Oncol. 2018;19:139-48.

7. Zhou C, Wu YL, Chen G, Feng J, Liu XQ, Wang C, et al. Final overall survival results from a randomised, phase III study of erlotinib versus chemotherapy as first-line treatment of EGFR mutation-positive advanced non-small-cell lung cancer (OPTIMAL, CTONG-0802). Ann Oncol. 2015;26: 1877-83.
See Article page 288.

\section{Commentary: Targeting our attention}

\section{Todd L. Demmy, MD, ${ }^{a}$ and Sai Yendamuri, $\mathrm{MBBS}^{\mathrm{b}}$}

The expert opinion article by Jones and colleagues ${ }^{1}$ is an extremely well-written essay that describes and provides context to the recently published ADAURA trial, which demonstrated dramatic improvements in survival for patients with stage IB-IIIA epidermal growth factor receptor mutation-positive non-small cell lung cancer who received adjuvant osimertinib. ${ }^{2}$ Because of its clarity and efficiency presenting key results and background, we have little to add and recommend it highly as a review article.

Instead, we wish to emphasize how this article portends a front of rapidly changing paradigms for our specialty for

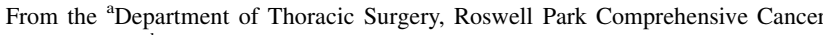
Center; and ${ }^{\mathrm{b}}$ Department of Surgery, University at Buffalo, Buffalo, NY.

Disclosures: T.L.D. reported Medtronic consultant. S.Y. reported no conflicts of interest.

The Journal policy requires editors and reviewers to disclose conflicts of interest and to decline handling or reviewing manuscripts for which they may have a conflict of interest. The editors and reviewers of this article have no conflicts of interest.

Received for publication Feb 11, 2021; revisions received Feb 11, 2021; accepted for publication Feb 12, 2021; available ahead of print Feb 24, 2021.

Address for reprints: Todd L. Demmy, MD, Department of Thoracic Surgery, Roswell Park Comprehensive Cancer Center, Elm and Carlton Sts, Buffalo, NY 14263 (E-mail: todd.demmy@roswellpark.org).

J Thorac Cardiovasc Surg 2021;162:294-5

$0022-5223 / \$ 36.00$

Copyright (c) 2021 by The American Association for Thoracic Surgery

http://dx.doi.org/10.1016/j.jtcvs.2021.02.069
}

Check for updates

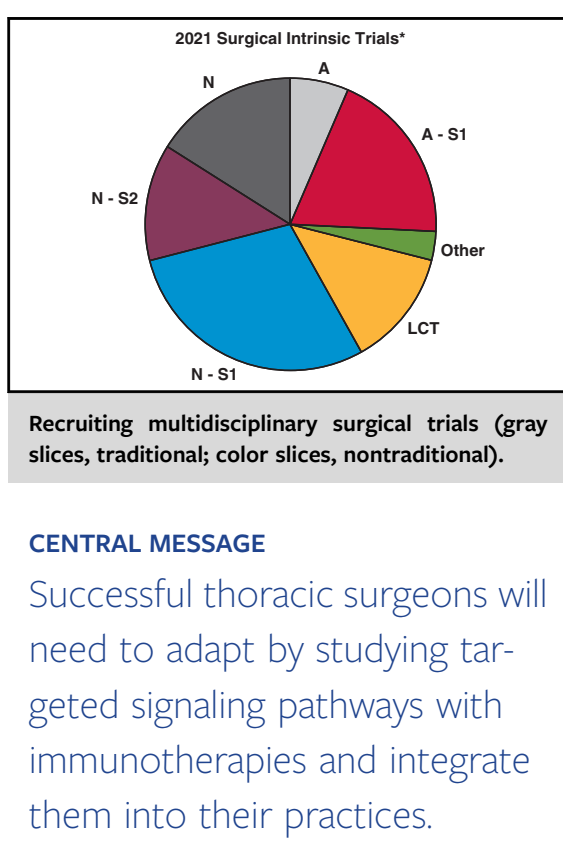

which we need to prepare. A review of current protocols open for enrollment reveals an explosion of new studies using novel treatment combinations with different sequencing in resectable non-small cell lung cancer. Figure 1 summarizes this review, with the gray slices representing studies that push eligibility to stages beyond that traditionally covered under clinical treatment guidelines. Some trials use a neoadjuvant approach $(\mathrm{N})$, whereas others use an 